\title{
Liliequist Membrane: Three- dimensional Constructive Interference in Steady State MR Imaging
}

\section{$\operatorname{AUTHOR}(\mathrm{S})$ :}

Fushimi, Yasutaka; Miki, Yukio; Ueba, Tetsuya; Kanagaki, Mitsunori; Takahashi, Takahiro; Yamamoto, Akira; Haque, Tabassum L.; ... Takahashi, Jun A.; Hashimoto, Nobuo; Konishi, Junji

\section{CITATION:}

Fushimi, Yasutaka ... [et al]. Liliequist Membrane: Three-dimensional Constructive Interference in Steady State MR Imaging. Radiology 2003, 229(2): 360-365

\section{ISSUE DATE:}

2003-11

URL:

http://hdl.handle.net/2433/218967

RIGHT:

(C) RSNA, 2003 


\section{Neuroradiology}

\section{Yasutaka Fushimi, MD \\ Yukio Miki, MD, PhD Tetsuya Ueba, MD, PhD Mitsunori Kanagaki, MD Takahiro Takahashi, MD Akira Yamamoto, MD Tabassum L. Haque, MBBS Junya Konishi, MD Jun A. Takahashi, MD, PhD Nobuo Hashimoto, MD, PhD Junji Konishi, MD, PhD \\ Index terms: \\ Brain, MR, 18.121412 \\ Brain, anatomy \\ Magnetic resonance (MR), pulse sequences, 18.121412 \\ Published online 10.1148/radiol.2292021507 Radiology 2003; 229:360-365 \\ Abbreviations: \\ CISS = constructive interference in steady state \\ CSF $=$ cerebrospinal fluid \\ $3 \mathrm{D}=$ three-dimensional}

\footnotetext{
${ }^{1}$ From the Departments of Nuclear Medicine and Diagnostic Imaging (Y.F., Y.M., M.K., T.T., A.Y., T.L.H., Junji Konishi) and Neurosurgery (T.U., J.A.T., N.H.), Kyoto University Graduate School of Medicine, 54 Shogoin Kawahara-cho, Sakyo-ku, Kyoto 606-8507, Japan; and Department of Radiology, Kobe University Graduate School of Medicine, Japan (Junya Konishi). Received November 19, 2002; revision requested January 21, 2003; revision received February 19, accepted March 13. Address correspondence to Y.M. (e-mail: mikiy $@ k u h p . k y o t o-u . a c . j p)$.

See also the commentary by Ward following this article.

Author contributions:

Guarantors of integrity of entire study, all authors; study concepts, Y.M., T.U., J.A.T., N.H.; study design, Y.F., Y.M., M.K., T.T., A.Y.; literature research, Y.F., Y.M.; clinical studies, Y.F., Y.M., M.K.; data acquisition, Y.F., Y.M., T.L.H., Junya Konishi, Junji Konishi; data analysis/interpretation, Y.F., Y.M., M.K., T.T., A.Y., T.L.H., Junya Konishi; manuscript preparation, Y.F., Y.M., T.U., J.A.T., N.H.; manuscript definition of intellectual content, Y.F., Y.M., T.U., M.K., T.T., A.Y., T.L.H., Junya Konishi, Junji Konishi; manuscript editing, revision/review, and final version approval, all authors

(c) RSNA, 2003
}

\section{Liliequist Membrane: Three-dimensional Constructive Interference in Steady State MR Imaging ${ }^{1}$}

\begin{abstract}
PURPOSE: To evaluate the Liliequist membrane in healthy volunteers by using three-dimensional (3D) Fourier transformation constructive interference in steady state (CISS) magnetic resonance (MR) imaging.

MATERIALS AND METHODS: In 31 volunteers, the authors performed 3D CISS MR imaging. They divided the membrane into three segments: the sellar, diencephalic, and mesencephalic segments. MR images were evaluated to identify the segments, superior and inferior attachments, lateral border, and thickness of the Liliequist membrane.

RESULTS: Three-dimensional CISS MR imaging depicted the sellar, diencephalic, and mesencephalic segments of the Liliequist membrane in the sagittal plane in 25 (81\%), $16(52 \%)$, and five (16\%) of the 31 subjects, respectively. Transverse MR imaging depicted these segments in 24 (77\%), 10 (32\%), and two (6\%) subjects, respectively, and coronal MR imaging depicted them in 24 (77\%), 12 (39\%), and two (6\%) subjects, respectively. Clear attachment of the membrane to the dorsum sellae was observed in 22 (88\%) of 25 subjects in whom the sellar segment was identified. Clear attachment to the mamillary body was identified in eight (50\%) of 16 subjects in whom the diencephalic segment was identified. The Liliequist membrane was attached to the oculomotor nerve on seven (14\%) of 50 sides of the lateral border and to the arachnoid membrane around the oculomotor nerve on 28 (56\%) sides. In the sagittal plane, the thickness of the membrane was less than one-half the thickness of the third ventricle floor in 22 (88\%) of 25 subjects.
\end{abstract}

CONCLUSION: The Liliequist membrane can be visualized by using 3D CISS MR sequences.

○ RSNA, 2003

The Liliequist membrane was described by Key and Retzius (1) in 1875. In 1956, Liliequist $(2,3)$ rediscovered the structure in his pneumoencephalographic studies of cadavers, which revealed this arachnoid membrane to be arising from the dorsum sellae to the anterior edge of the mamillary body. He also reported that during pneumoencephalography, the presence of this membrane initially caused the air to remain in the interpeduncular cistern before it gradually filled the chiasmatic cistern. Other authors subsequently described the Liliequist membrane on the basis of postmortem study results (4-7).

With the development of modern endoscopic techniques for neurovascular procedures, it has become possible to treat hydrocephalus endoscopically. Endoscopic third ventriculostomy, one of the most sophisticated of these techniques, is now widely performed because it is minimally invasive $(8-12)$. With endoscopic third ventriculostomy, the floor of the third ventricle is fenestrated; then, the flow of cerebrospinal fluid (CSF) is diverted from the third ventricle to the subarachnoid space. Some authors have reported failures of this procedure $(13,14)$. Buxton et al $(13)$ proposed that the Liliequist membrane, which is not fenestrated during endoscopic third ventriculostomy, may have blocked the flow of CSF from the third ventricle.

The Liliequist membrane is also known to partially or completely obstruct CSF flow at the suprasellar cistern. It is also closely associated with the formation of the suprasellar 
arachnoid cyst $(15,16)$. Pulsations in CSF and increased pressure below the obstructing membrane result in upward expansion of the membrane to form a cystic diverticulum.

Although the importance of the Liliequist membrane has been emphasized, some discrepancies regarding the location of this structure exist. There is agreement in the literature on the lower attachment of this membrane to the dorsum sellae. However, there is disagreement concerning the site of the superior attachment-namely, whether this attachment is premamillary $(3,5,17)$ or retromamillary $(4,7)$. This discordance may be attributable to variation in the sites of attachment. Similarly, conflicting descriptions of the lateral border of the Liliequist membrane have been reported: Some have reported that the lateral edge of the membrane attaches to the arachnoidal sheath surrounding the oculomotor nerve (4), whereas others have described the lateral margins as attaching to the pia mater covering the temporal uncus $(5,7)$ or the tentorium (6).

In our review of the literature, the Liliequist membrane has been identified in many neuroanatomic studies, but in most of these, the described findings have been from cadaveric investigations, and few studies have involved examination of the membrane in living individuals. As far as magnetic resonance (MR) imaging studies are concerned, to our knowledge, only one case report has described the Liliequist membrane as assessed with MR imaging (13).

It is well known that neuroanatomic evaluations of the cisterns and surrounding structures are complicated. Three-dimensional (3D) Fourier transformation constructive interference in steady state (CISS) enables high-spatial-resolution refocused gradient-echo MR imaging. Three-dimensional CISS MR imaging depicts small structures surrounded by CSF with high contrast and high spatial resolution and is suitable for MR cisternography. Cranial nerves III (18), IV (19), VI $(18,20)$, VII $(21)$, VIII $(21,22)$, and IX-XII (23) have been successfully visualized on MR images obtained by using 3D CISS sequences. This sequence has also been applied to virtual endoscopy of the CSF space (24). The Liliequist membrane has been described as a fine arachnoid membrane in the subarachnoid space. Therefore, these reported successful visualizations of the cranial nerves led us to use 3D CISS MR sequences to delineate the anatomic course of the Liliequist membrane. The purpose of our study was to evaluate the Liliequist membrane in healthy volunteers by using 3D CISS MR imaging.

\section{MATERIALS AND METHODS}

MR imaging examinations were performed in 31 healthy volunteers (19 men aged 24-41 years [mean age, 30.2 years] and 12 women aged $24-53$ years [mean age, 32.3 years]). The criterion for volunteer recruitment was age between 20 and 60 years. All subjects were neurologically examined by one neurosurgeon (T.U.) and received a diagnosis of neurologically healthy. No subjects demonstrated neurologic symptoms, which also were evaluated by T.U. The local ethics committee at Kyoto University Graduate School of Medicine approved the study, and all participants provided written informed consent before entering the study.

\section{MR Imaging}

All MR imaging examinations were performed with a 1.5-T unit (Magnetom Symphony; Siemens, Erlangen, Germany) by using a regular head coil. A 3D CISS pulse MR sequence was used with the following parameters: 12.30/6.15 (repetition time msec/echo time msec), $70^{\circ}$ flip angle, $160 \times 160-\mathrm{mm}$ (read $\times$ phase encode) field of view, 70.0-mm slab thickness, $256 \times$ 256 matrix, 52 3D partitions, one slab, $0.6 \times 0.6-\mathrm{mm}$ pixel size, $0.6-\mathrm{mm}$ effective section thickness, one signal acquired, and imaging time of 8 minutes 10 seconds.

\section{Image Analysis}

Data obtained with 3D CISS MR sequences were reconstructed in sagittal, transverse, and coronal planes with a section thickness of $0.6 \mathrm{~mm}$. Images were analyzed with a multiplanar reconstruction program (Siemens), which enabled us to compare the position of any point selected in one plane with the position of the same point in the other two perpendicular planes simultaneously. Two experienced neuroradiologists (Y.F. with 6 years experience, Y.M. with 17 years experience) analyzed the images collaboratively. Each neuroradiologist made initial evaluations independently, and any disagreements regarding final conclusions were resolved by consensus between the two neuroradiologists.

\section{Anatomic Course and Identification of the Liliequist Membrane}

We identified the Liliequist membrane by using the following criterion: a thin (ie, $\leq 1 \mathrm{~mm}$ ) structure behind the infun-

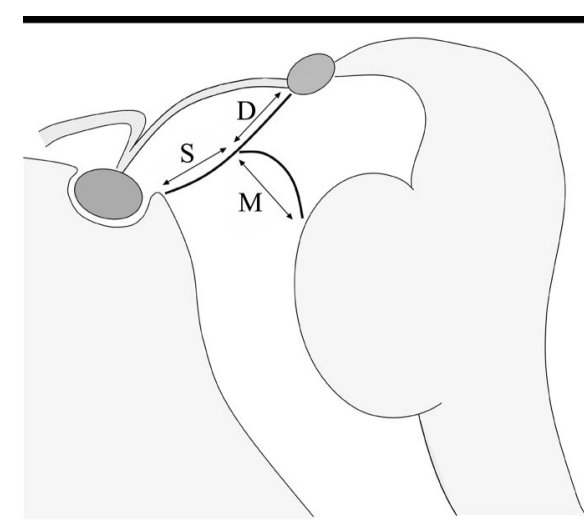

Figure 1. Schematic illustration of the Liliequist membrane in the sagittal plane. $D=$ diencephalic segment, $M=$ mesencephalic segment, $S=$ sellar segment.

dibulum, below the floor of the third ventricle, and in front of the basilar artery recognized on more than three consecutive image sections. Next, we divided the membrane into three segments and named them the sellar, diencephalic, and mesencephalic segments (Fig 1). These three segments corresponded to the microanatomy of the Liliequist membrane described in a previous report (4). The identification of the Liliequist membrane was assessed by using a three-score scale: A score of 2 meant a positive identification; a score of 1; a highly probable identification; and a score of 0 , no identification.

Attachments of the Liliequist membrane to the dorsum sellae and the mamillary body were assessed in the sagittal plane. When there was clear continuation of the membrane from the dorsum sellae or from the mamillary body, the membrane was described as demonstrating clear attachment. When continuity was ill defined, this was defined as obscure attachment. No continuity was defined as absent attachment.

We also assessed each side of the lateral border of the Liliequist membrane in the coronal and transverse planes. We defined the structure to which the membrane first attached laterally as the lateral border. When the lateral border was not identified, it was described as such.

Finally, we visually assessed the thickness of the Liliequist membrane at its thickest part in the sagittal plane and compared it with the thickness of the third ventricle floor, because this floor can always be identified near the membrane and the thickness of this floor seems to differ infrequently among individuals. Membrane thickness was described as follows: less than one-half the thickness of the floor, less than the thick- 


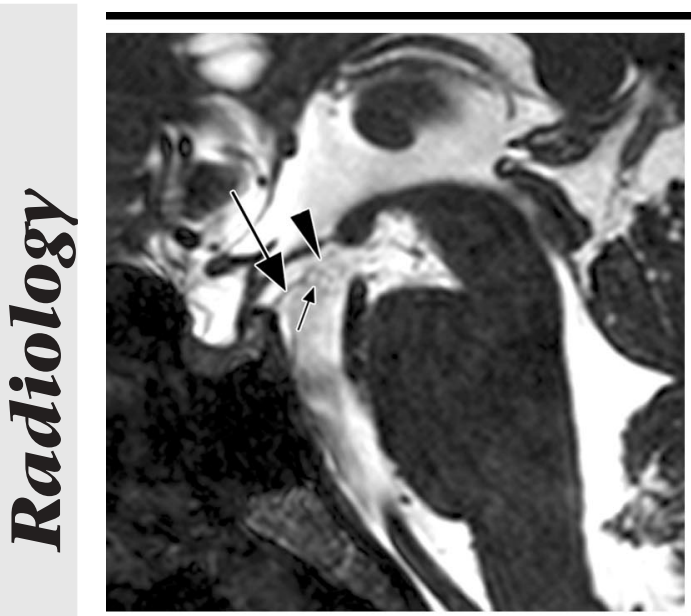

a.

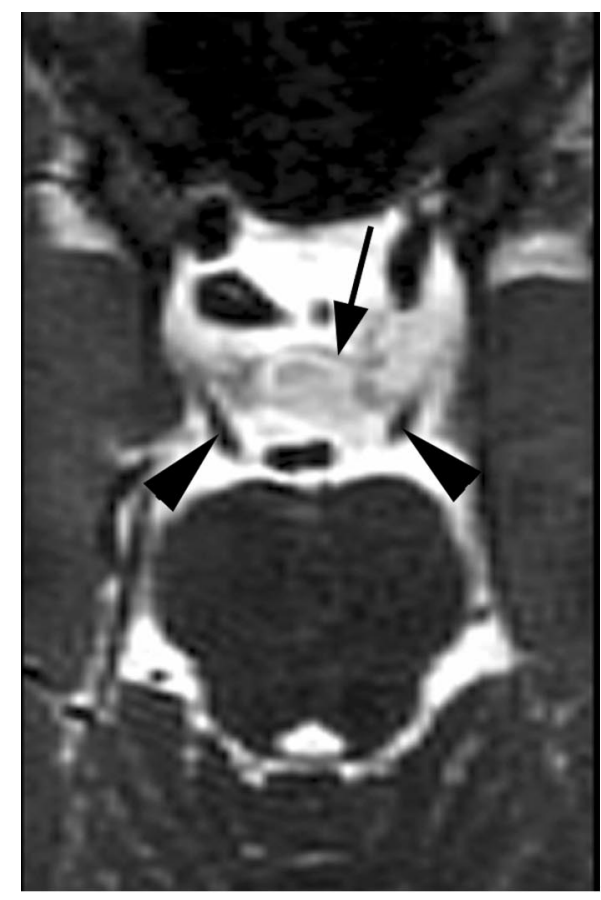

d.

ness of the floor but greater than or equal to one-half the thickness of the floor, or greater than or equal to the thickness of the floor.

\section{RESULTS}

The three segments of the Liliequist membrane (ie, sellar, diencephalic, and mesencephalic segments) were analyzed in the 31 subjects by using CISS MR sequences (Fig 2). In the sagittal plane, the sellar, diencephalic, and mesencephalic segments were identified (score of 1 or 2) in $25(81 \%), 16(52 \%)$, and five (16\%) of the 31 subjects, respectively (Table 1$)$. In the transverse plane, these segments

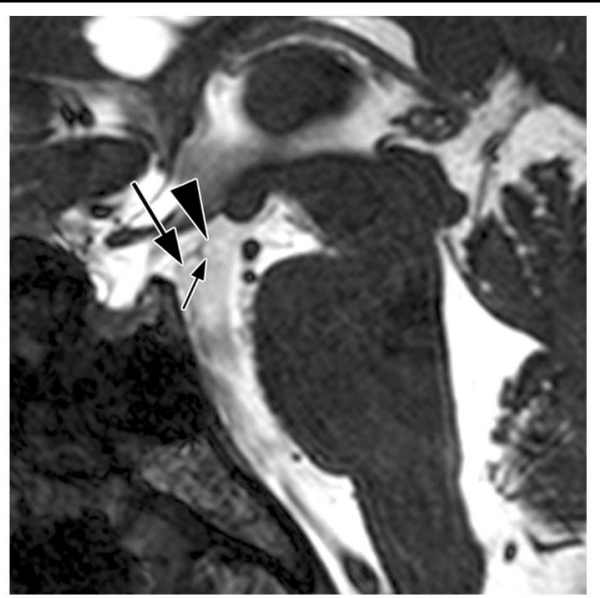

b.

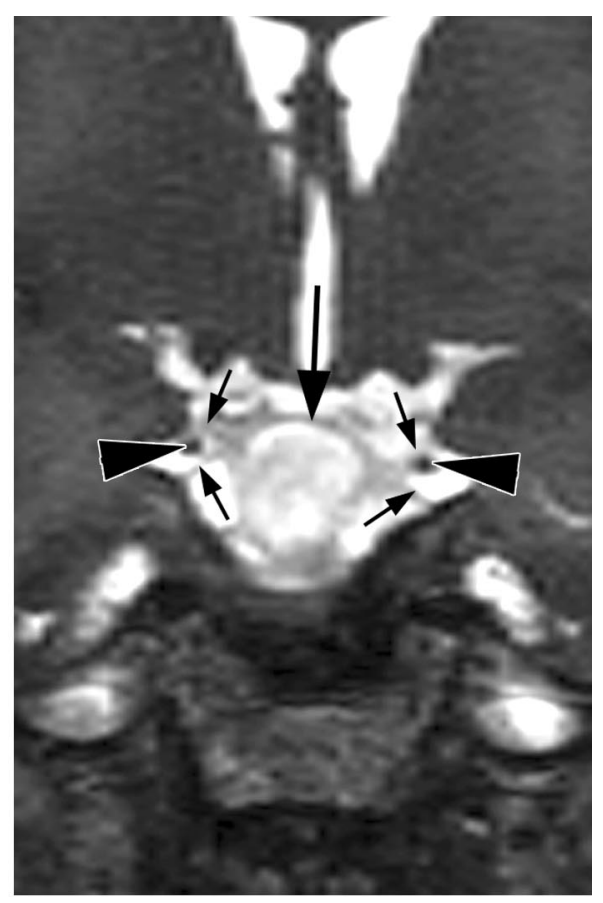

e.

were identified in 24 (77\%), 10 (32\%), and two (6\%) subjects, respectively (Table 1). In the coronal plane, the segments were identified in 24 (77\%), 12 (39\%), and two (6\%) subjects, respectively (Table 1). When the Liliequist membrane was visualized, the sellar segment was always identified. In six individuals (19\%), no segment of the membrane was visualized.

At MR examination in the 25 subjects in whom the sellar segment was identified in the sagittal plane, clear attachment to the dorsum sellae was observed in 22 subjects; obscure attachment, in two; and absent attachment, in one (Table 2). Among the 16 subjects with a recognizable diencephalic segment in the sagittal plane, clear attachment to the

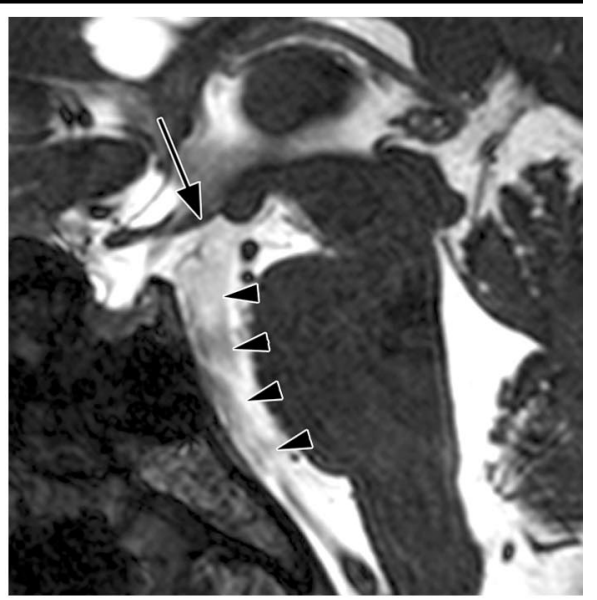

c.

Figure 2. CISS MR images (12.30/6.15) obtained in a 30-year-old man. (a, b) Sagittal images obtained at (a) median and (b) right paramedian levels show the sellar segment (large arrow), diencephalic segment (arrowhead), and tip of the mesencephalic segment (small arrow) of the Liliequist membrane. The point at which the diencephalic segment attaches to the mamillary body is obscure. (c) Sagittal image obtained at the same level as b shows a CSF flow artifact (arrowheads) in the prepontine and interpeduncular cisterns that is blocked by the Liliequist membrane. The thickness of the Liliequist membrane is less than one-half the thickness of the third ventricle floor (arrow). (d) Transverse image depicts the sellar segment (arrow) and the oculomotor nerves (arrowheads) bilaterally. (e) Coronal image depicts the sellar segment (large arrow) and the lateral borders of the Liliequist membrane attached bilaterally to the arachnoid membranes (small arrows) around both oculomotor nerves (arrowheads).

mamillary body was identified in eight subjects (Table 2), and in all eight of them, the diencephalic segment was attached to the front aspect of the mamillary body. Obscure and absent attachments to the mamillary body were seen in seven subjects and one subject, respectively (Table 2).

In 25 subjects, the Liliequist membrane could be identified in the coronal or transverse planes-that is, some segments of this membrane were recognizable (score of 1 or 2 ) in these planes. The right and left sides of the lateral border of the Liliequist membrane were assessed in these 25 subjects (50 sides) (Table 3 ). On three right sides and four left sides, the membrane directly attached to the oculomotor nerve (Fig 3, Table 3). On 15 right sides and 13 left sides, direct attachment was not confirmed; rather, the membrane appeared to be localized around the oculomotor nerve (Fig 4, Table 3). On seven right sides and eight left 
TABLE 1

Identification of Sellar, Diencephalic, and Mesencephalic Segments of Liliequist Membrane in 31 Subjects

\begin{tabular}{|c|c|c|c|c|c|c|c|c|c|}
\hline \multirow{2}{*}{$\begin{array}{l}\text { Identification } \\
\text { Score* }^{*}\end{array}$} & \multicolumn{3}{|c|}{ Sagittal Plane } & \multicolumn{3}{|c|}{ Transverse Plane } & \multicolumn{3}{|c|}{ Coronal Plane } \\
\hline & Sellar & Diencephalic & Mesencephalic & Sellar & Diencephalic & Mesencephalic & Sellar & Diencephalic & Mesencephalic \\
\hline 2 & $\begin{array}{c}22 \\
(71)\end{array}$ & $\begin{array}{c}10 \\
(32)\end{array}$ & $\begin{array}{c}2 \\
(6)\end{array}$ & $\begin{array}{c}21 \\
(68)\end{array}$ & $\begin{array}{c}9 \\
(29)\end{array}$ & 0 & $\begin{array}{c}21 \\
(68)\end{array}$ & $\begin{array}{c}9 \\
(29)\end{array}$ & 0 \\
\hline 1 & 3 & $\begin{array}{c}6 \\
(19)\end{array}$ & 3 & 3 & $\begin{array}{c}1 \\
\text { (3) }\end{array}$ & 2 & 3 & 3 & $\begin{array}{c}2 \\
(6)\end{array}$ \\
\hline 0 & $\begin{array}{c}6 \\
(19)\end{array}$ & $\begin{array}{l}15 \\
(48)\end{array}$ & $\begin{array}{c}26 \\
(84)\end{array}$ & $\begin{array}{c}7 \\
(23)\end{array}$ & $\begin{array}{c}21 \\
(68)\end{array}$ & $\begin{array}{l}29 \\
(94)\end{array}$ & $\begin{array}{c}7 \\
(23)\end{array}$ & $\begin{array}{l}19 \\
(61)\end{array}$ & $\begin{array}{l}29 \\
(94)\end{array}$ \\
\hline
\end{tabular}

Note.-Data are numbers of segments identified in the given planes. Numbers in parentheses are percentages.

* Score definitions: 2 = positive identification, $1=$ highly probable identification, $0=$ no identification.

sides, the lateral border of the Liliequist membrane was unidentifiable (Table 3).

As previously mentioned, the Liliequist membrane in 25 subjects was visualized in the sagittal plane-that is, some segments of this membrane were recognizable (score of 1 or 2) in this plane. In 22 of these 25 subjects, the thickness of the membrane in the sagittal plane was less than one-half the thickness of the third ventricle floor. In three subjects, the thickness of the membrane was less than that of the floor but either greater than or equal to one-half the floor's thickness (Fig 5). No membranes had a thickness greater than or equal to the thickness of the third ventricular floor (Table 4).

\section{DISCUSSION}

With the development of microsurgery and the introduction of endoscopic techniques to intracranial surgery, the Liliequist membrane has come to be considered an important structure. However, to our knowledge, there have been no studies of the MR imaging assessment of the Liliequist membrane in healthy living individuals.

Of the three segments of the Liliequist membrane, the sellar segment was the most frequently identified at 3D CISS MR imaging. This segment was identified in 25 subjects. The diencephalic segment was identified in 16 subjects and the mesencephalic segment in only five. Since the mesencephalic membrane is thinner than the diencephalic membrane and frequently incomplete and contains an opening through which the basilar artery ascends (4), it may be difficult to identify the mesencephalic segment on MR images. Zhang and An (6) reported that 43\% of the 35 cadavers that they examined did not have identifiable diencephalic or mesencephalic components of the Liliequist membrane. Our study results partly support this finding: We identified the diencephalic and mesencephalic segments

\section{TABLE 2 \\ Attachment of Liliequist Membrane Segments to Dorsum Sellae and Mamillary Body}

\begin{tabular}{ccc}
\hline Depiction of Attachment & Dorsum Sellae Attachment & Mamillary Body Attachment \\
\hline Clear & $22(88)$ & $8(50)$ \\
Obscure & $2(8)$ & $7(44)$ \\
Absent & $1(4)$ & $1(6)$ \\
All & 25 & 16 \\
\hline
\end{tabular}

Note.-Data are numbers of sellar and diencephalic segments identified (score of 1 or 2 ) in the sagittal planes to be attached to the given structures. Numbers in parentheses are percentages.

\section{TABLE 3}

Attachment of Lateral Border of Liliequist Membrane to Oculomotor Nerve

\begin{tabular}{lrr}
\hline \multicolumn{1}{c}{ Type of Attachment } & Right & Left \\
\hline Directly to oculomotor nerve & $3(12)$ & $4(16)$ \\
Around oculomotor nerve & $15(60)$ & $13(52)$ \\
Lateral border not identified & $7(28)$ & $8(32)$ \\
\cline { 2 - 4 } All & 25 & 25 \\
\hline
\end{tabular}

Note.-Data are numbers of sides of the Liliequist membrane identified (score of 1 or 2 ) in the coronal or transverse planes. Numbers in parentheses are percentages.

in at least five subjects (16\%) at 3D CISS MR imaging. Both of these components were less frequently detected in the present study, probably because the mesencephalic membrane was thinner, incomplete, or fenestrated and was difficult to visualize with $3 \mathrm{D}$ CISS MR imaging. Likewise, regarding the segments that were not identified at MR imaging (score of 0 ), in some cases, the segment might have existed but could not be depicted for the same reason.

Disagreements regarding the superior attachment of the Liliequist membrane exist in the literature. Our data support the idea of a premamillary attachment, although some exceptions might exist for subjects with unidentifiable (score of 0) diencephalic segments at MR imaging. Our study data reconfirmed that the in- ferior attachment of the membrane is the dorsum sellae.

The lateral margins of the Liliequist membrane were observed directly attaching to the oculomotor nerve on seven of the 50 sides identified with 3D CISS MR imaging. On another 28 sides, the lateral margins were close to the oculomotor nerve; these findings indicate that the arachnoid membrane around the oculomotor nerve connected with the Liliequist membrane. Matsuno et al (4) reported that the lateral edges of the Liliequist membrane were attached to the arachnoidal sheath surrounding the oculomotor nerve. Brasil and Schneider (5) documented that the lateral borders of the membrane were above the oculomotor nerve and attached laterally to the pia of the mesial surface of the temporal un- 


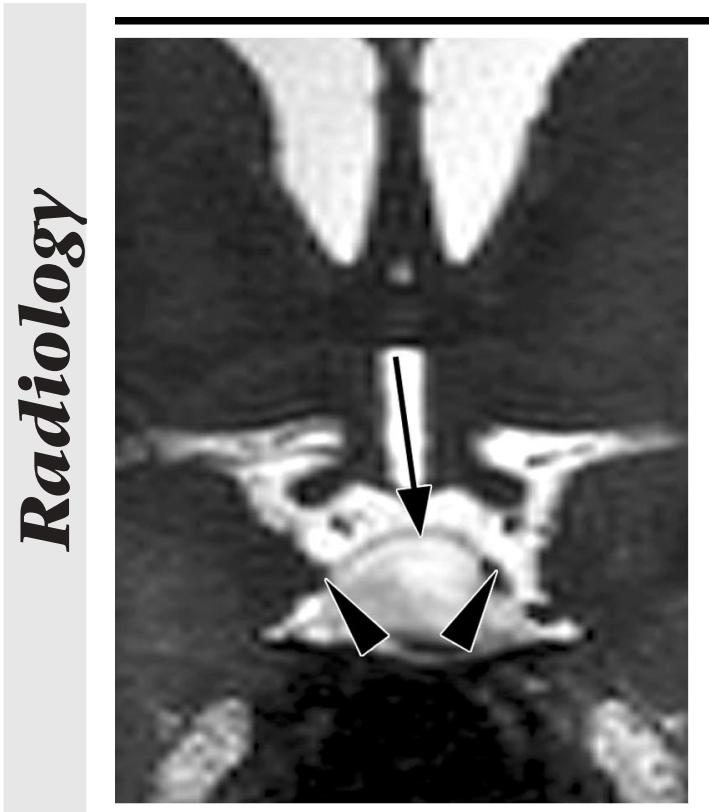

a.

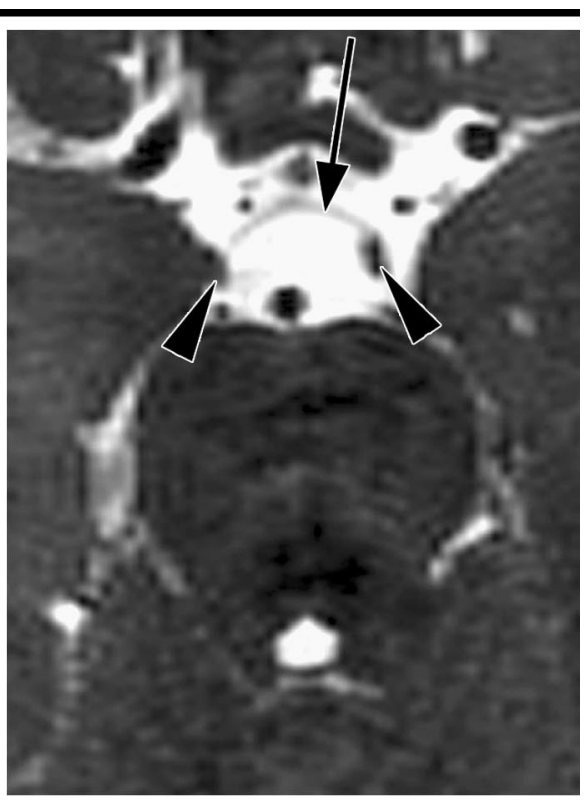

b.

Figure 3. (a) Coronal and (b) transverse CISS MR images (12.30/6.15) obtained in a 28-year-old woman show the lateral border of the Liliequist membrane (arrow). The membrane is directly attached to the oculomotor nerves (arrowheads) bilaterally.

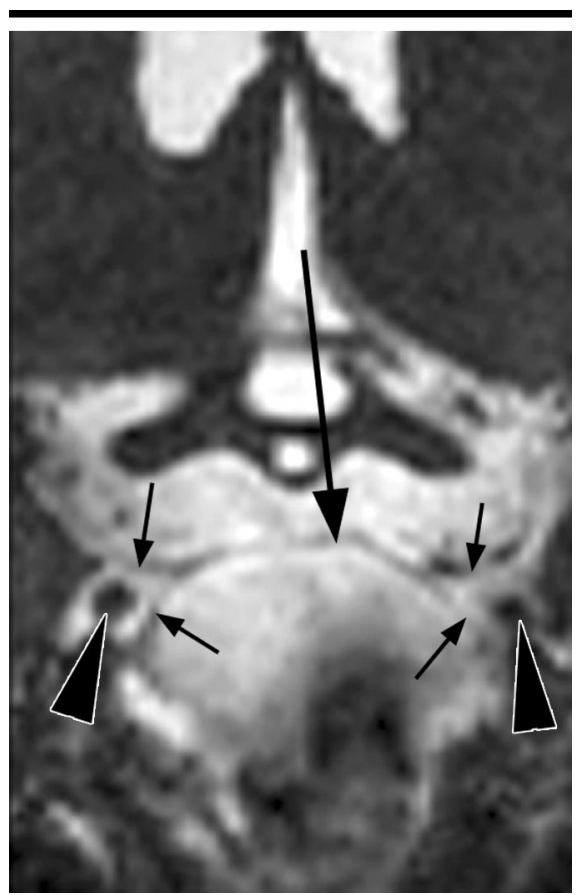

Figure 4. Coronal CISS MR image (12.30/ 6.15) obtained in a 34-year-old man shows the Liliequist membrane (large arrow) and the arachnoid membrane (small arrows) converging around the oculomotor nerves (arrowheads).

cus. Vinas and Panigrahi (7) reported that the lateral margins of the Liliequist membrane were attached to the pia on the mesial surface of the temporal uncus. Zhang and An (6) observed the Liliequist

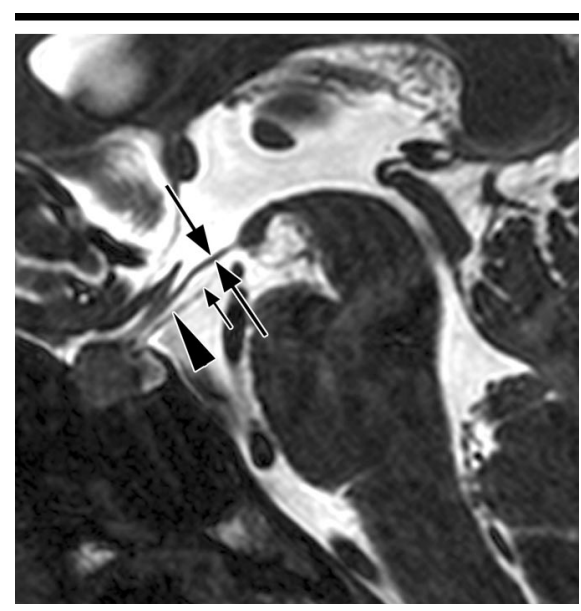

Figure 5. Sagittal CISS MR image (12.30/ 6.15) obtained in a 30-year-old woman shows the sellar (arrowhead) and diencephalic (small arrow) segments. The mesencephalic segment is not depicted. The thickness of the sellar segment is less than the thickness of the third ventricle floor (large arrows) but greater than one-half the thickness of the floor.

membrane extending laterally and continuing with the tentorium above and below the tentorial edge. They commented, however, that the arachnoid trabecular network has very wide connections with surrounding structures and that borders of the irregular network may vary widely among individuals.

Our results support the contention that the Liliequist membrane attaches to the oculomotor nerve or to the arach-
TABLE 4

Thickness of Liliequist Membrane in Sagittal Plane

\begin{tabular}{cc}
\hline Thickness* & No. of Membranes \\
\hline$<1 / 2$ Floor & $22(88)$ \\
$\geq 1 / 2$ Floor & $3(12)$ \\
\cline { 2 - 2 } All & 25 \\
\hline
\end{tabular}

Note.-The thickest segment of the membrane in the sagittal plane was compared with the thickness of the floor of the third ventricle. Numbers in parentheses are percentages.

$*<1 / 2$ floor $=$ less than one-half the thickness of the floor, $\geq 1 / 2$ floor $=$ thickness less than the thickness of the floor but greater than or equal to one-half the thickness of the floor.

noid membrane surrounding the nerve, but this membrane may well extend further laterally beyond the oculomotor nerve. We could not identify the lateral borders of 15 sides, probably because the cisternal anatomy is too complicated to visualize at MR imaging.

In the present study, 3D CISS MR sequences played an important role in the identification of the Liliequist membrane and its relationship with the oculomotor nerve. CSF artifacts existed to varying degrees but did not impede visualization of the Liliequist membrane in most subjects. Since the Liliequist membrane is a cisternal structure, the 3D CISS MR sequence was quite useful in this study.

The Liliequist membrane is the arachnoid membrane dividing the chiasmatic and interpeduncular cisterns. The predominant amount of CSF is known to flow through the ventral surface of the brain stem to the interpeduncular cistern. When complete, the Liliequist membrane may block CSF flow from the interpeduncular cistern. Without perforation of the Liliequist membrane, third ventriculostomy may fail and further interventions to perforate the membrane may be necessary (13). Before performing CSF diversion procedures such as endoscopic third ventriculostomy, determining whether the Liliequist membrane is complete may prove useful. Future pre- and postoperative studies may reveal the clinical usefulness of CISS MR imaging in endoscopic third ventriculostomy. Present study results show that the Liliequist membrane can be visualized in most healthy subjects by using 3D CISS MR sequences.

Acknowledgments: The authors are grateful for the help and technical assistance of Ari Kobayashi, RT, and Akira Hiraga, RT. 


\section{References}

1. Key A, Retzius G. Studien in der anatomie des nervensystems und des bindegewebs. Stockholm, Sweden: Norstedt \& Soner, $1875 ; 111-123$.

2. Liliequist B. The anatomy of the subarachnoid cisterns. Acta Radiol 1956; 46: 61-71.

3. Liliequist B. The subarachnoid cisterns: an anatomic and roentgenologic study. Acta Radiol 1959; 185:S1-S108.

4. Matsuno H, Rhoton AL Jr, Peace D. Microsurgical anatomy of the posterior fossa cisterns. Neurosurgery 1988; 23:58-80.

5. Brasil AV, Schneider FL. Anatomy of Liliequist membrane. Neurosurgery 1993; 32:956-960.

6. Zhang M, An PC. Liliequist's membrane is a fold of the arachnoid mater: study using sheet plastination and scanning electron microscopy. Neurosurgery 2000; 47:902-908.

7. Vinas FC, Panigrahi M. Microsurgical anatomy of the Liliequist membrane and surrounding neurovascular territories. Minim Invasive Neurosurg 2001; 44:104-109.

8. Grunert P, Perneczky A, Resch K. Endoscopic procedures through the foramen interventriculare of Monro under stereotactical conditions. Minim Invasive Neurosurg 1994; 37:2-8.

9. Brockmeyer D, Abtin K, Carey L, Walker ML. Endoscopic third ventriculostomy: an outcome analysis. Pediatr Neurosurg 1998; 28:236-240.

10. Cinalli G, Salazar C, Mallucci C, Yada JZ, Zerah M, Sainte-Rose C. The role of endo- scopic third ventriculostomy in the management of shunt malfunction. Neurosurgery $1998 ; 43: 1323-1327$.

11. Hopf NJ, Grunert P, Fries G, Resch KD, Perneczky A. Endoscopic third ventriculostomy: outcome analysis of 100 consecutive procedures. Neurosurgery 1999; 44 : 795-804.

12. Bergsneider M, Holly LT, Lee JH, King WA, Frazee JG. Endoscopic management of cysticercal cysts within the lateral and third ventricles. J Neurosurg 2000; 92:14-23.

13. Buxton N, Vloeberghs M, Punt J. Liliequist membrane in minimally invasive endoscopic neurosurgery. Clin Anat 1998; 11:187-190.

14. Weaver KD, Ewend MG. Development of an arachnoid cyst after ventriculoperitoneal shunt placement: case illustration. J Neurosurg 2000; 92:363.

15. Binitie O, Williams B, Case CP. A suprasellar subarachnoid pouch; aetiological considerations. J Neurol Neurosurg Psychiatry 1984; 47:1066-1074.

16. Miyajima M, Arai H, Okuda O, Hishii $M$, Nakanishi H, Sato K. Possible origin of suprasellar arachnoid cysts: neuroimaging and neurosurgical observations in nine cases. J Neurosurg 2000; 93:62-67.

17. Yasargil MG. Microneurosurgery: microsurgical anatomy of the basal cisterns and vessels of the brain. Stuttgart, Germany: Georg Thieme, 1984; 5-54.

18. Held P, Nitz W, Seitz J, et al. Comparison of 2D and 3D MRI of the optic and oculomotor nerve anatomy. Clin Imaging 2000; 24:337-343.
19. Yousry I, Moriggl B, Dieterich M, Naidich TP, Schmid UD, Yousry TA. MR anatomy of the proximal cisternal segment of the trochlear nerve: neurovascular relationships and landmarks. Radiology 2002; 223:31-38.

20. Yousry I, Camelio S, Wiesmann M, et al. Detailed magnetic resonance imaging anatomy of the cisternal segment of the abducent nerve: Dorello's canal and neurovascular relationships and landmarks. J Neurosurg 1999; 91:276-283.

21. Held P, Fellner C, Fellner F, et al. MRI of inner ear and facial nerve pathology using 3D MP-RAGE and 3D CISS sequences. Br J Radiol 1997; 70:558-566.

22. Mateijsen DJ, Van Hengel PW, Krikke AP, Van Huffelen WM, Wit HP, Albers FW. Three-dimensional Fourier transformation constructive interference in steady state magnetic resonance imaging of the inner ear in patients with unilateral and bilateral Meniere's disease. Otol Neurotol 2002; 23:208-213.

23. Seitz J, Held P, Frund R, et al. Visualization of the IXth to XIIth cranial nerves using 3-dimensional constructive interference in steady state, 3-dimensional magnetizationprepared rapid gradient echo and T2weighted 2-dimensional turbo spin echo magnetic resonance imaging sequences. J Neuroimaging 2001; 11:160-164.

24. Shigematsu Y, Korogi Y, Hirai T, et al. Virtual MRI endoscopy of the intracranial cerebrospinal fluid spaces. Neuroradiology $1998 ; 40: 644-650$.

\section{monewa no \\ Clinician's Commentary ${ }^{1}$}

Fushimi et al (1) have performed an elegant study of the anatomic variations of the membrane of Liliequist. They have rightfully noted the potential importance that this anatomic variation may have in the success or failure of endoscopic third ventriculostomy (ETV).

The overall success rate of ETV is around $75 \%(2-4)$. However, this leaves a population of patients in whom the procedure does not work. The obvious question is why? Several studies have tried to address this question (4-7).

In performing ETV, the intent is to perforate the floor of the third ventricle. When "failures" of ETV are analyzed, the emphasis is usually on whether there was a patent stoma of the third ventricle (7). In one study in which the potential failures of an ETV procedure were assessed, a $36 \%$ variation of the anatomy, with a thickened floor of the third ventricle being the most common, was reported (6). No mention was made of the anatomic variations of the membrane of Liliequist. Another study, focusing on imaging correlates of successful ETV, concentrated on ventricular size and a flow void in cine flow studies.

It is interesting that $75 \%$ of unsuccessful ETV procedures involve an absence of a flow void, but there is no mention of where the blockage existed. The presumption is that it was at the level of the floor of the third ventricle (5). In another study, repeat endoscopy was performed in the cases of failed ETV; a patent third ventricle perforation was present in seven of 12 patients (8). Whether the membrane of Liliequist had a role in these failed procedures is unknown. However, as the results of the present study by Fushimi et al (1) indicate, failure to assess the patency of the membrane of Liliequist may be an important factor that needs to be addressed in the future.

\section{References}

1. Fushimi Y, Miki Y, Ueba T, et al. Liliequist Membrane: three-dimensional constructive interference in steady state MR imaging. Radiology 2003; 229:360-365.

2. Brockmeyer D, Abtin K, Carey L, Walker ML. Endoscopic third ventriculostomy: an outcome analysis. Pediatric Neurosurgery 1998; 28:236-240.

3. Hopf NJ, Grunert P, Fries G, Resch KD,
Perneczky A. Endoscopic third ventriculostomy: outcome analysis of 100 consecutive procedures. Neurosurgery 1999; 44:795-804.

4. Javadpour M, Mallucci C, Brodbelt A, Golash A, May P. The impact of endoscopic third ventriculostomy on the management of newly diagnosed hydrocephalus in infants. Pediatric Neurosurgery 2001; 35:131-135.

5. Kulkarni AV, Drake JM, Armstrong DC, Dirks PB. Imaging correlates of successful endoscopic third ventriculostomy. J Neurosurg 2000; 92:915-919.

6. Rohde V, Gilsbach JM. Anomalies and variants of the endoscopic anatomy for third ventriculostomy. Minimum Invasive Neurosurgery 2000; 43:111-117.

7. Fukuhara T, Luciano MG, Kowalski RJ. Clinical features of third ventriculostomy failures classified by fenestration patency. Surgical Neurology 2002; 58:102-110.

8. Mohanty A, Vasudev MK, Sampath S, Radhesh S, Sastry Kolluri VR. Failed endoscopic third ventriculostomy in children management options. Pediatric Neurosurgery 2002; 37:304-309.

${ }^{1}$ Department of Surgery, Virginia Commonwealth Univeristy, School of Medicine PO Box 980631, Richmond, VA 23298 e-mail: jdward@vcu.edu 